\title{
Survive an innate immune response through XBP1
}

\author{
Arthur Kaser ${ }^{1}$, Richard S Blumberg ${ }^{2}$ \\ ${ }^{1}$ Department of Medicine II, Innsbruck Medical University, Anichstrasse 35, A-6020 Innsbruck, Austria; ${ }^{2}$ Division of Gastroen- \\ terology, Hepatology, and Endoscopy, Department of Medicine, Brigham and Women's Hospital, Harvard Medical School, 75 \\ Francis Street, Boston MA 02115, USA
}

Cell Research (2010) 20:506-507. doi:10.1038/cr.2010.61; published online 3 May 2010

Endoplasmic reticulum (ER) stress occurs upon the accumulation of unfolded or misfolded proteins, and induces adaptive mechanisms, termed the unfolded protein response (UPR), aimed at resolving ER stress and hence preventing potentially dysfunctional proteins from impairing proper cell function [1]. The UPR in general is an evolutionary highly conserved mechanism, with one branch in particular (the IRE1-XBP1 axis) conserved from yeast to metazoans including man. While highly secretory cells are particularly dependent upon an efficient UPR, it is also obvious that a multitude of secondary factors that affect protein folding may lead to the accumulation of misfolded proteins and hence ER stress; these factors include the metabolic state of the cell, oxygen supply, redox state, and many others [1].

An intriguing study by Richardson et al. now reports that innate immune activation is a potent trigger of ER stress in Caenorhabditis elegans ( $C$. elegans), with the XBP1-dependent branch of the UPR being required to recover from and survive the stress associated with the elicitation of an in-

Correspondence: Arthur Kaser ${ }^{\text {a }}$, Richard S

Blumberg $^{\mathrm{b}}$

${ }^{\mathrm{a}}$ Tel: +43 51250481996

E-mail: arthur.kaser@i-med.ac.at

${ }^{\mathrm{b}} \mathrm{Tel}$ : +1-617-732-6917

E-mail: rblumberg@partners.org nate immune response [2]. Specifically, infection with Pseudomonas aeruginosa (P. aeruginosa) induced ER stress secondary to activation of PMK-1 [2], the p38 mitogen-activated protein kinase (MAPK) orthologue which had previously been shown to be essential for resistance against microbial pathogens [3]. In contrast to wild-type larvae, loss-offunction $x b p-1$ mutants exhibited larval lethality upon $P$. aeruginosa infection, which was associated with disruption of ER morphology. Quite surprisingly, larval lethality did not occur in the absence of p38 PMK-1. However, overactivation of PMK-1 in the absence of pathogenic bacteria via silencing of $v h p-1$, a MAPK phosphatase, in the context of loss-offunction $x b p-1$ mutants recapitulated the lethal phenotype described above [2]. $x b p-1$ did not affect the rate of accumulation of $P$. aeruginosa, excluding the possibility that developmental lethality upon $x b p-1$ loss-of-function is attributable to accelerated infection [2]. Moreover, loss-of-function in the two other branches of the UPR (atf-6 and pek-1) had no effect on larval development in the presence of $P$. aeruginosa [2], identifying the IRE1-XBP1 axis as the critical protective UPR branch induced secondary to an innate immune response.

XBP1 (Hac1 in yeast) is activated by IRE1 via an unconventional splicing mechanism that leads to a frame-shift in XBP1 mRNA and hence translation of the active UPR transcription factor XBP1s. Infection of C. elegans with $P$. aeruginosa induces the transcription of more than 300 genes, with $50 \%$ predicted to involve processing in the ER [4]. The occurrence of $x b p-1$ splicing secondary to $P$. aeruginosa infection was not only dependent on pmk-1, but also on the transcription factor atf-7, since an atf-7 mutant abrogated the pathogen-induced expression of genes regulated by the PMK-1 pathway [2]. Since tunicamycin-induced ER stress and hence $x b p-1$ splicing was not impaired in pmk-1 mutants, ER stress induction upon $P$. aeruginosa infection appears to be unique and dependent upon a PMK-1-dependent orchestration of a transcriptional program that is involved in the innate immune response to the pathogen. It remains to be determined how PMK-1 links to IRE1 as this is the only known endoribonucelase that is capable of generating transcriptionally active, spliced $x b p-1$.

Using a reporter system monitoring hsp4 (the C. elegans orthologue of mammalian grp78/BiP) induction, ER stress upon $P$. aeruginosa infection localized to the intestine, the site of infection, and coincided with increased $x b p-1$ splicing [2]. It is interesting, therefore, that hypomorphic XBP1 and unresolved ER stress in intestinal epithelial cells (IECs) has recently been linked to intestinal inflammation in mice, and polymorphisms in $X B P 1$ have 
been associated with both forms of human inflammatory bowel disease (IBD), Crohn's disease (CD) and ulcerative colitis (UC) [5]. In that mouse model, IECs with hypomorphic Xbpl function exhibited increased responsiveness towards inflammatory and microbial stimuli, and the cell types that were most severely affected were Paneth cells and goblet cells, both of which are highly secretory cells which differentiate from intestinal epithelial stem cells. Paneth cells, located at the base of intestinal crypts, secrete abundant antimicrobial peptides that affect the composition of the intestinal microbiota [6], while goblet cells produce mucins. The notion that innate immune activation may induce ER stress and indeed requires an efficient XBP1-branch of the UPR to let the organism cope with innate immune activation supports the view that even minor impairments in UPR function might have substantial effects for the development of immune-mediated diseases. In this context it is noteworthy that in addition to polymorphisms in $X B P 1$, polymorphisms in the protein disulfide isomerase $A G R 2$ have been linked with CD and UC [7]. AGR2 is implicated in ER protein folding, and Agr2 $2^{-\leftarrow}$ mice exhibit evidence of ER stress and disruption in goblet and Paneth cell homeostasis [8]. In the context of the genetic $X B P 1$ association with IBD and the evolutionarily ancient role of $x b p-1$ and ER stress with innate immune function, it will be important to investigate whether the intestinal microbiota, or perhaps even specific pathogenic microbes [9], might be at the basis of induction of ER stress in the IEC compartment and consequently intestinal inflammation that may arise from this if hypomorphic. Apart from its role in IEC function, the known requirement for proper XBP1 function in dendritic cell function in mice might serve as another evolutionarily conserved example wherein the mechanisms established in C. elegans might have an important role in mammalian innate immune defense as well [10].

Another interesting angle of ER stress induction by activation of innate immune pathways and its dependency on a proper UPR relates to the recent report that TLR signaling induces selective suppression of the ATF4CHOP branch of the UPR. Specifically, prior TLR engagement prevented the phospho-eIF $2 \alpha$ promotion of ATF 4 translation, a potent inducer of $\mathrm{CHOP}$ during ER stress [11]. Administration of the TLR4 agonist LPS accordingly prevented apoptosis of macrophages, hepatocytes, and renal tubule cells during systemic ER stress via a TRIFdependent pathway [11]. It was suggested that this mechanism might have evolved to support the survival of TLR-expressing cells (e.g. innate immune cells) that encounter ER stress during the host response to invading pathogens [11]. Hence, these studies together suggest that at least two of the three arms of the UPR (IRE1-XBP1 and PERK-EIF2-ATF4) support the ability of innate immune cells to prevail through the stress associated with a response to a pathogen.

In summary, the study by Richardson et al. expands the universe of physiological pathways that are dependent upon a proper UPR by demonstrating a key involvement of $x b p-1$ in the consequences associated with an innate immune response. The IRE1-XBP1 branch of the UPR has previously been shown to be critically involved in plasma cell differentiation [12] and hence the adaptive immune system as well. It appears that the IRE1-XBP1 pathway is not only the evolutionarily most conserved branch of the UPR, but evolutionarily far more deeply involved in immune responses to pathogens than previously anticipated. These observations further highlight the substantial amount of cellular stress that the host experiences in interacting with the foreign hostile environment posed by invading pathogens and potentially commensal microorganisms within mucosal tissues.

\section{References}

1 Ron D, Walter P. Signal integration in the endoplasmic reticulum unfolded protein response. Nat Rev Mol Cell Biol 2007; 8:519-529.

2 Richardson CE, Kooistra T, Kim DH. An essential role for XBP-1 in host protection against immune activation in C. elegans. Nature 2010; 463:10921095.

3 Kim DH, Feinbaum R, Alloing G, et al. A conserved p38 MAP kinase pathway in Caenorhabditis elegans innate immunity. Science 2002; 297:623-626.

4 Troemel ER, Chu SW, Reinke V, Lee SS, Ausubel FM, Kim DH. p38 MAPK regulates expression of immune response genes and contributes to longevity in C. elegans. PLoS Genet 2006; 2:e183.

5 Kaser A, Lee AH, Franke A, et al. XBP1 links ER stress to intestinal inflammation and confers genetic risk for human inflammatory bowel disease. Cell 2008; 134:743-756.

6 Salzman NH, Hung K, Haribhai D, et al. Enteric defensins are essential regulators of intestinal microbial ecology. Nat Immunol 2010; 11:76-83.

7 Zheng W, Rosenstiel P, Huse K, et al. Evaluation of AGR2 and AGR3 as candidate genes for inflammatory bowel disease. Genes Immun 2006; 7:11-18.

8 Zhao F, Edwards R, Dizon D, et al. Disruption of Paneth and goblet cell homeostasis and increased endoplasmic reticulum stress in Agr $2^{-/-}$mice. Dev Biol 2010; 338:270-279.

9 Zhang FR, Huang W, Chen SM, et al. Genomewide association study of leprosy. $N$ Engl J Med 2009; 361:26092618.

10 Iwakoshi NN, Pypaert M, Glimcher LH. The transcription factor XBP-1 is essential for the development and survival of dendritic cells. $J$ Exp Med 2007; 204:2267-2275.

11 Woo CW, Cui D, Arellano J, et al. Adaptive suppression of the ATF4-CHOP branch of the unfolded protein response by toll-like receptor signalling. Nat Cell Biol 2009; 11:1473-1480.

12 Iwakoshi NN, Lee AH, Vallabhajosyula P, Otipoby KL, Rajewsky K, Glimcher LH. Plasma cell differentiation and the unfolded protein response intersect at the transcription factor XBP-1. Nat Immunol 2003; 4:321-329. 